\section{Relacionamento probabilístico: recuperação de informações de óbitos infantis e natimortos em localidade no Maranhão, Brasil}

\author{
Probabilistic record linkage: recovery of data on \\ infant deaths and stillbirths in Maranhão State, \\ Brazil
}

\author{
1 Secretaria Municipal de \\ Saúde de São Luís, São Luís, \\ Brasil. \\ 2 Secretaria de Estado da \\ Saúde do Maranhão, São \\ Luís, Brasil. \\ 3 Programa de Pós-graduação \\ em Saúde Coletiva, \\ Universidade Federal do \\ Maranhão, São Luís, Brasil. \\ 4 Departamento de Medicina \\ III, Universidade Federal do \\ Maranhão, São Luís, Brasil. \\ 5 Hospital Universitário, \\ Universidade Federal do \\ Maranhão, São Luís, Brasil. \\ ${ }_{6}$ Departamento de Saúde \\ Pública, Universidade \\ Federal do Maranhão, São \\ Luís, Brasil. \\ 7 Departamento de Medicina \\ I, Universidade Federal do \\ Maranhão, São Luís, Brasil. \\ Correspondência \\ R. A. A. Rafael \\ Programa de Pós-graduação \\ em Saúde Coletiva, \\ Universidade Federal do \\ Maranhão. \\ Rua Barão de Itapary 155, São \\ Luís, MA 65020-070, Brasil. \\ rrafael@globo.com
}

Roberio Antonio Alencar Rafael 1,2,3 Valdinar Sousa Ribeiro 3,4 Milady Cutrim Vieira Cavalcante 1,3,5 Alcione Miranda dos Santos 6 Vanda Maria Ferreira Simões 5,7

\section{Introdução}

This study used probabilistic record linkage to estimate underreporting of stillbirths and infant deaths in Maranhão State, Northeast Brazil, in 2008. Records were linked between the Hospital Information System $(S I H)(N=374,418)$ and the Mortality Information System (SIM) ( $N=26,597)$, using data extraction, identification, and processing software. Under-recording rates were 9.7\% for stillbirths, $12 \%$ for neonatal deaths, and 5.3\% for post-neonatal deaths. In the larger municipalities, the correction of the infant mortality rate was greater than 19\%. The superiority of information on infant deaths in the SIH (as compared to the SIM) was $6.5 \%$ in municipalities with $<25$ thousand inhabitants, $2 \%$ in municipalities with 25 80 thousand inhabitants, and $8.3 \%$ in those with $>80$ thousand inhabitants. Inconsistencies involved non-completion of items/variables, discordant data on the same event, and in some cases even total absence of death records. The method proved useful for retrieving mortality data for the SIM from the SIH.

Underregistration; Information Systems; Mortality
Alguns problemas limitam as análises de mortalidade infantil no Brasil. A ocorrência de sub-registro de óbitos gerais referentes ao ano 2007 alcança percentuais diferenciados nas várias Unidades da Federação, sendo praticamente inexistente no Rio de Janeiro e Rio Grande do Sul, mas alcança 41\% no Amapá e 43\% no Maranhão 1.

Este evento tem magnitude expressiva nas regiões Norte e Nordeste 2 . No Estado do Maranhão, a cobertura média de óbitos gerais variou $36,4 \%$ em 2000 para 57,2\% em 2007 3, uma das mais baixas do país. Destaca-se que as informações para óbitos infantis não estavam disponíveis, mas, habitualmente estes apresentam maior sub-registro que os óbitos gerais 3 .

O Sistema de Informações sobre Mortalidade (SIM) é a maior fonte de dados para notificações de óbitos. Em estudo comparando dados do SIM com o Sistema de Informações Hospitalares (SIH), demonstrou-se que os estados da Região Nordeste apresentavam mais óbitos captados por este, ocorrendo o oposto nas regiões Sul e Sudeste 4. Desse modo, observou-se no Maranhão maior cobertura de óbitos entre natimortos no SIH (314\%) e no período de 7-27 dias (110\%); no Ceará a taxa de mortalidade neonatal precoce estimada pelo SIH foi seis vezes maior que a calculada pelo SIM 4 .

Constitui possível causa do sub-registro de óbitos a ocorrência de sepultamentos sem a exi- 
gência da certidão, nos denominados cemitérios clandestinos, sendo associada à pobreza, especialmente na área rural 5 . Fatores ligados ao registro do evento também são identificados como associados à subnotificação, tais como problemas no fluxo da Declaração de Óbito (DO), quer por extravio ou por negligência na alimentação das bases de dados, deixando o órgão central sem a informação. Tal situação faz com que os gestores desconheçam o real perfil epidemiológico, interferindo negativamente nas decisões para a alocação de recursos, definição de estratégias e ações mais eficazes de políticas públicas para redução de óbitos infantis.

Em relação à Autorização de Internação Hospitalar (AIH), seu principal objetivo é de cobrança de procedimento. Desse modo, tem periodicidade de processamento e fluxo distinto da DO, não incluindo, como nesta, o setor epidemiológico e de estatísticas vitais das Secretarias de Saúde. Estima-se que $74 \%$ das internações no país, fonte de alimentação do SIH, ocorram em hospitais vinculados ao SUS ${ }^{3}$. No Estado do Maranhão este tipo de atendimento atinge $96 \%$ das internações ${ }^{3}$.

Desse modo, devido à sua ampla cobertura, o SIH é utilizado em diferentes estudos, incluindo análises econômicas em saúde 6 , estimativas de indicadores de mortalidade 4 , assistência hospitalar pública 7 e como instrumento de vigilância à saúde ${ }^{8}$. Sua utilização na recuperação de informações do SIM assume importância por proporcionar o conhecimento real dos níveis de mortalidade e da classificação dos óbitos.

O SIM e SIH apresentam objetivos específicos, vantagens e limitações que lhe são peculiares. No entanto, quando as bases são vinculadas, como com o uso da técnica de linkage, podem-se corrigir inconsistências nas variáveis, preencher lacunas e identificar os eventos não captados por uma das fontes, como demonstrado em alguns estudos 9,10,11.
Diante do exposto, reveste-se de especial importância o estudo de bases de dados diversas que possibilitem estimar com maior precisão a magnitude da mortalidade infantil e assegurar maior confiabilidade no acompanhamento temporal deste indicador. O presente estudo analisou os registros de óbitos do SIM e SIH para estimar o sub-registro de dados no sistema de mortalidade referentes ao ano de 2008, no Maranhão.

\section{Métodos}

Foi realizada análise dos bancos de dados do SIM e SIH em relação aos óbitos infantis e natimortos, acessados nos arquivos da Secretaria Estadual de Saúde do Maranhão.

Neste estudo foram incluídos registros de natimortos e de óbitos de crianças com idade inferior a um ano, residentes no Estado do Maranhão, constantes nos sistemas SIM e/ou SIH em 2008. Incluíram-se ainda informações de parturientes que apresentavam dados de natimorto ou de parto seguido de morte infantil contidos no SIH. Neste sistema, foram consideradas as internações das competências do ano de 2008 e dos quatro primeiros meses de 2009, mas referentes àquele. Tal recorte foi realizado devido ao prazo estendido de processamento da AIH. Não foram incluídos registros com dados ausentes no campo "nome da mãe" ou nos campos "data de nascimento" e "idade" simultaneamente. As AIH com parto único que apresentaram preenchimento simultâneo dos campos para nascido vivo e nascido morto, foram computadas como sendo de natimortos.

Os bancos com dados elegíveis para este estudo passaram por um processo de "deduplicação" a fim de eliminar registros duplicados. Para cálculo do sub-registro, inicialmente, os municípios do Maranhão foram agrupados conforme porte populacional baseado na classificação de Drumond et al. 12 (Tabela 1).

Tabela 1

Distribuição dos municípios de acordo com a população residente no Maranhão, Brasil, 2008.

\begin{tabular}{lcccc}
\hline Porte populacional do município (habitantes) & População & $\%$ & Municípios & \% \\
\hline$<25.000$ & 2.045 .620 & 32,44 & 155 & 71,43 \\
$25.001-80.000$ & 1.944 .452 & 30,84 & 50 & 12 \\
$>80.000$ & 2.315 .467 & 36,72 & 5,53 & 100,00 \\
Total & 6.305 .539 & 100,00 & 217 &
\end{tabular}

Fonte: Departamento de Informática do SUS (DATASUS; http://www.datasus.gov.br, acessado em 26/Jan/2010). 
Em seguida, por meio do processo de linkage, foi identificado o número de registros de natimortos e óbitos infantis do SIH que não possuíam correspondência no SIM.

O processo de linkage foi feito por meio do relacionamento probabilístico, dada a ausência de dados determinísticos como número do Cadastro de Pessoa Física (CPF), Cartão SUS (CadSUS) ou Registro Geral (RG). Utilizaram-se etapas sequenciais: padronização das bases de dados, relacionamento propriamente dito, subdividido em blocagem e pareamento, combinação dos arquivos e revisão manual 13 .

Para extração de dados do SIH foi utilizado o programa SIHDTS do Departamento de Informática do SUS (DATASUS). O programa Access (Microsoft Corp., Estados Unidos) serviu para selecionar as variáveis de interesse para este estudo e diminuir o tamanho dos campos destas no SIH e no SIM. A seguir empregou-se o programa RecLink III.

A padronização dos arquivos foi necessária em um único momento e envolveu a transformação dos caracteres não numéricos para letra maiúscula, a exclusão de acentos, a eliminação de preposições e sinais de pontuação erroneamente digitados. Também permitiu a formatação de datas em um padrão único e a subdivisão do campo "nome da mãe", cujas partes foram utilizadas na etapa seguinte de blocagem e de pareamento.

A blocagem consistiu na criação de blocos, ou seja, conjuntos comuns de dados dentro de arquivos que foram relacionados a fim de permitir que o processo de pareamento fosse feito de forma otimizada 13

As variáveis selecionadas para o relacionamento probabilístico foram de referência e complementares. O nome da mãe (primeiro e último) foi a variável de referência. Fez-se uso do soundex 13 utilizando-se três estratégias consecutivas de blocagem. Em primeiro lugar foi feita a blocagem pela combinação dos códigos soundex do último e do primeiro nome da mãe. No passo seguinte foi realizada a blocagem pelo soundex apenas do primeiro nome e por fim, usou-se a blocagem pelo soundex do último nome da mãe 14 .

Na comparação dos campos utilizou-se o algoritmo aproximado, adotando-se como parâmetros de configuração: $99 \%$ de sensibilidade, $2 \%$ de especificidade e proporção mínima de concordância de $75 \%$. O erro tipo I adotado foi de $5 \%$.

Quando encontradas dúvidas ou divergências na identificação do nome da mãe entre os dois sistemas foram consideradas as variáveis complementares: município de residência da mãe; data de nascimento, data e causa do óbito da criança.
Para os relacionamentos propriamente ditos (SIH e SIM) foram aplicados separadamente a cada um dos arquivos do SIH, de crianças e de parturientes.

A operação de combinação dos arquivos permitiu que se criasse um novo arquivo, com base no relacionamento, permitindo a seleção de campos dos arquivos de origem (SIM e SIH) que deveriam constar do arquivo final ${ }^{15}$. A seguir realizou-se revisão manual dos pares duvidosos, para confirmar se os registros do SIH tinham ou não correspondência no SIM (Figura 1).

O coeficiente de mortalidade neonatal foi calculado a partir da divisão do número de óbitos neonatais (recém-nascidos que morreram até o 27o dia) pelo número de nascidos vivos, por mil; de mortalidade pós-neonatal a partir da divisão dos óbitos pós-neonatais (ocorridos entre 28 dias e um dia antes de completar um ano) pelo número de nascidos vivos, por mil; e de mortalidade infantil da divisão de óbitos ocorridos entre o nascimento até um dia antes de completar um ano de idade, pelo número de nascidos vivos, por mil. O coeficiente de natimortalidade (CNM) foi definido pela divisão do número de natimortos (com idade gestacional $>22$ semanas) pela soma do número de nascidos vivos e nascidos mortos, por mil 16. Foram considerados os registros de nascidos vivos residentes no Maranhão em 2008 que constavam no Sistema de Informações sobre Nascidos Vivos (SINASC).

No cálculo dos sub-registros foram quantificados para cada categoria (natimortos, óbitos neonatais e pós-neonatais) os registros que estavam no SIH sem correspondência no SIM, denominado registros recuperados pelo SIH (RRSIH) e calculada sua porcentagem, através da seguinte equação:

$\%$ RRSIH $=[$ RRSIH $/($ SIM + RRSIH $)] * 100$

Também foram calculados o CNM, Coeficiente de Mortalidade Neonatal (CMN), Coeficiente de Mortalidade Pós-Neonatal (CMPN) e o Coeficiente de Mortalidade Infantil (CMI) considerando os dados constantes no SIM, primeiramente e, em seguida, a soma destes com as informações recuperadas pelo SIH, denominado SIM corrigido (SIMC).

\section{Resultados}

O banco das declarações de óbito do SIM no Maranhão contém 2.052 registros de óbitos infantis (1.387 neonatais e 665 pós-neonatais) e 1.530 registros de natimortos. Dentre os 374.418 
Fluxograma do relacionamento das bases de dados Sistema de Informações sobre Mortalidade (SIM) e Sistema de Internações Hospitalares (SIH).

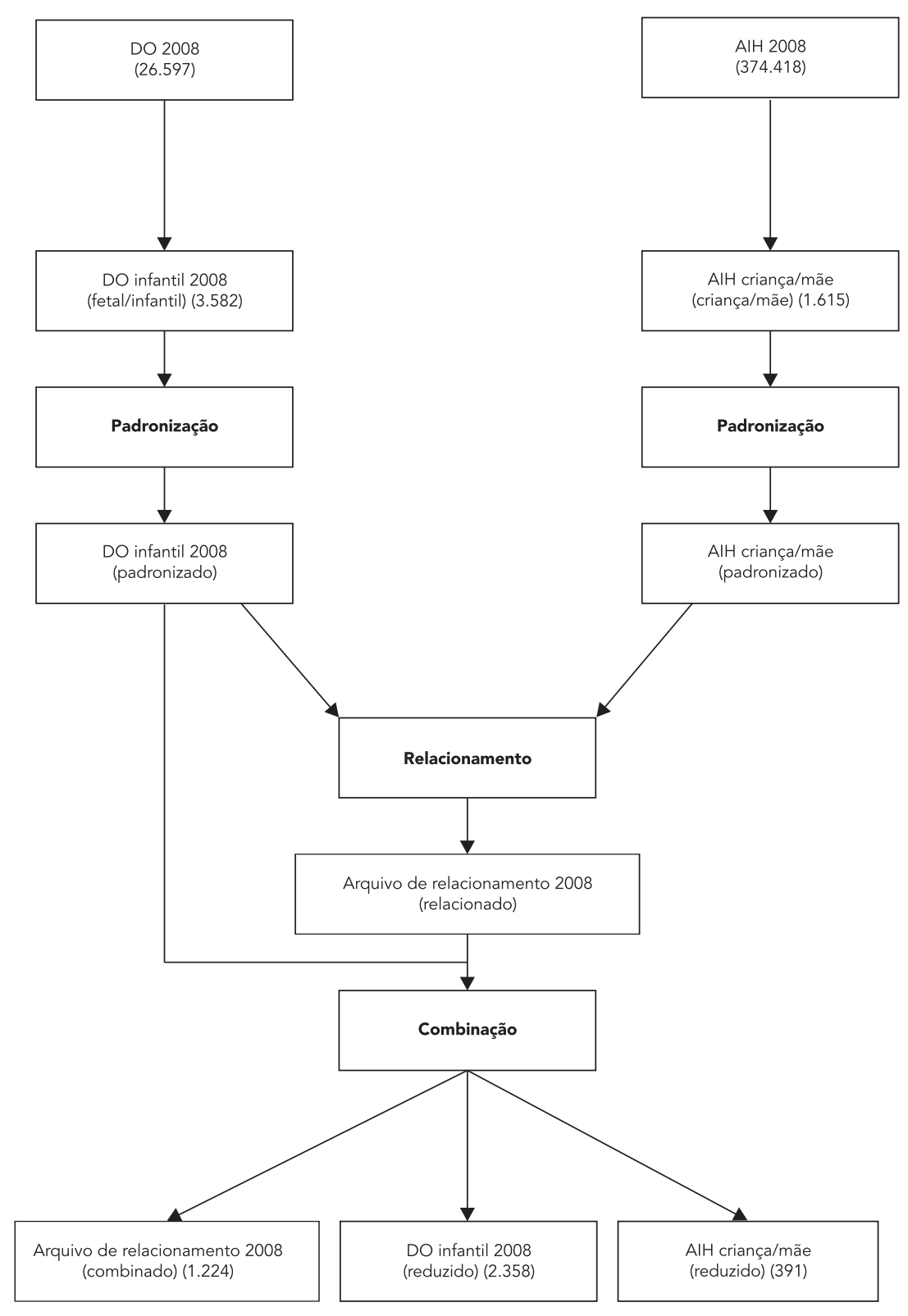

AlH: Autorização de Internação Hospitalar; DO: Declaração de Óbito.

registros de AIH verificaram-se 685 óbitos neonatais, 144 pós-neonatais e 786 de natimortos. Não foram incluídos neste estudo 9 registros devido ausência de preenchimento dos campos "data de nascimento" e "idade" simultaneamente e 13, por ausência no campo "nome da mãe”.
Os arquivos originais do SIM e SIH contendo apenas informações relativas aos óbitos infantis e aos natimortos apresentaram 391 registros do SIH não relacionáveis ao SIM. Foram identificadas no SIM, informações incompletas/ausentes nos campos "nome da mãe" (17), "data de nas- 
cimento" (15), "data do registro" (145), "nome do médico" (145), "sexo" (54 foram indeterminados), "idade gestacional" (25), assim como erros que sugerem duplicidade da DO.

Observou-se ainda que 169 registros de óbitos infantis $(8,2 \%)$ tinham causas mal definidas. Em relação ao SIH estavam incorretas as informações relativas aos campos "nascido vivo" e "nascido morto" (331 registros simultâneos, equivalente a $20,5 \%$ das AIHs do estudo), e diferenças no período de internação da AIH e data do óbito na DO. É importante destacar a ausência de registro de natimortos (SIM e SIH) em 25 municípios e de óbitos infantis (SIM e SIH) em 13 municípios, o que pode ser devido ou à ausência de assistência, ou ao eventual atendimento fora do domicílio de residência, seguido de equívoco no preenchimento da DO ou da AIH.

Após o relacionamento dos arquivos, o SIH apresentou informações desconhecidas pelo
SIM relacionadas aos natimortos (9,7\%), aos óbitos neonatais (12\%) e aos pós-neonatais $(5,3 \%)$ (Tabela 2). Ressalta-se que em municípios com mais de 80 mil habitantes, cerca de $20 \%$ dos óbitos neonatais foram recuperados pelo $\mathrm{SIH}$.

Também nos municípios com mais de $80 \mathrm{mil}$ habitantes o CMN observado no SIM (12,8 por mil) foi corrigido para 16,0 por mil (SIMC), acréscimo de $24,9 \%$, o CMI (18,0 por mil) foi corrigido para 21,6 por mil, acréscimo de 19,8\% (Tabela 3 ). Já o coeficiente de natimortalidade foi corrigido em menor proporção, variando de 13,7 por mil para 15 por mil, acréscimo de $9,2 \%$ (Tabela 3 ).

O sub-registro de natimortos no SIM em relação ao SIH foi de 15,5\% nos municípios com menos de 25 mil habitantes e chegou a $20 \%$ nos municípios de 20 a 80 mil habitantes. Em relação aos óbitos infantis, foi maior nos municípios menores $(6,5 \%)$ que nas intermediárias $(2 \%)$ (Tabela 4).

Tabela 2

Informações sobre natimortos, óbitos infantis e identificação do sub-registro do Sistema de Informações sobre Mortalidade (SIM) em relação ao Sistema de Informações Hospitalares (SIH). Maranhão, Brasil, 2008.

\begin{tabular}{|c|c|c|c|c|c|c|c|c|c|c|c|c|}
\hline \multirow{3}{*}{$\begin{array}{l}\text { Porte populacional do } \\
\text { município (habitantes) }\end{array}$} & \multicolumn{4}{|c|}{ Natimorto } & \multicolumn{4}{|c|}{ Óbito neonatal } & \multicolumn{4}{|c|}{ Óbito pós-neonatal } \\
\hline & \multirow{2}{*}{$\begin{array}{l}\text { SIM } \\
n\end{array}$} & \multirow{2}{*}{$\begin{array}{c}\mathrm{SIH} \\
\mathrm{n}\end{array}$} & \multicolumn{2}{|c|}{ RRSIH } & \multirow{2}{*}{$\begin{array}{l}\text { SIM } \\
n\end{array}$} & \multirow{2}{*}{$\begin{array}{c}\mathrm{SIH} \\
\mathrm{n}\end{array}$} & \multicolumn{2}{|c|}{ RRSIH } & \multirow{2}{*}{$\begin{array}{c}\text { SIM } \\
\mathrm{n}\end{array}$} & \multirow{2}{*}{$\begin{array}{c}\mathrm{SIH} \\
\mathrm{n}\end{array}$} & \multicolumn{2}{|c|}{ RRSIH } \\
\hline & & & $\mathbf{n}$ & $\%$ & & & $\mathbf{n}$ & $\%$ & & & $\mathrm{n}$ & $\%$ \\
\hline$<25.000(n=155)$ & 455 & 236 & 55 & 10,8 & 362 & 130 & 27 & 6,9 & 224 & 42 & 10 & 4,3 \\
\hline $25.000-80.000(n=50)$ & 443 & 225 & 51 & 10,3 & 443 & 112 & 17 & 3,7 & 206 & 39 & 10 & 4,6 \\
\hline$>80.000(n=12)$ & 632 & 325 & 59 & 8,5 & 582 & 443 & 145 & 19,9 & 235 & 63 & 17 & 6,7 \\
\hline Total & 1.530 & 786 & 165 & 9,7 & 1.387 & 685 & 189 & 12,0 & 665 & 144 & 37 & 5,3 \\
\hline
\end{tabular}

RRSIH: registros recuperados pelo $\mathrm{SIH}$.

Tabela 3

Coeficientes de natimortalidade (CNM), mortalidade neonatal (CMN), pós-neoanatal (CMPN) e infantil (CMI), segundo dados do Sistema de Informações sobre Mortalidade (SIM) e do Sistema de Informações sobre Mortalidade Corrigido (SIMC).

Maranhão, Brasil, 2008.

\begin{tabular}{lcccccccc}
\hline $\begin{array}{l}\text { Porte populacional do } \\
\text { município (habitantes) }\end{array}$ & \multicolumn{2}{c}{ CNM (\%) } & \multicolumn{2}{c}{ CMN (\%) } & \multicolumn{2}{c}{ CMPN (\%) } & \multicolumn{2}{c}{ CMI (\%) } \\
& SIM & SIMC & SIM & SIMC & SIM & SIMC & SIM & SIMC \\
\hline \multirow{2}{*}{$25.000(\mathrm{n}=155)$} & 11,5 & 12,9 & 9,3 & 10,0 & 5,7 & 6,0 & $15,1 *$ & 15,9 \\
$25.000-80.000(n=50)$ & 10,7 & 11,9 & 10,8 & 11,2 & 5,0 & 5,3 & 15,8 & 16,5 \\
$>80.000(n=12)$ & 13,7 & 15,0 & 12,8 & 16,0 & 5,2 & 5,6 & 18,0 & 21,6 \\
Total & 12,0 & 13,3 & 11,1 & 12,6 & 5,3 & 5,6 & 16,4 & 18,2 \\
\hline
\end{tabular}

* Considerou três casos de óbitos infantis sem especificação da faixa etária. 
Tabela 4

Frequência de municípios com sub-registro no Sistema de Informações sobre Mortalidade (SIM) de natimortos e óbitos infantis segundo o porte populacional. Maranhão, Brasil, 2008.

\begin{tabular}{lcccc}
\hline $\begin{array}{l}\text { Porte populacional do município } \\
\text { (habitantes) }\end{array}$ & \multicolumn{4}{c}{ Informações do SIH > SIM } \\
& Natimortos & Óbitos infantis \\
\hline$<25.000(n=155)$ & 24 & 15,5 & 10 & $\%$ \\
$25.000-80.000(n=50)$ & 10 & 20,0 & 1 & 2,0 \\
$>80.000(n=12)$ & 2 & 16,7 & 1 & 8,3 \\
Total & 36 & 16,6 & 12 & 5,5 \\
\hline
\end{tabular}

SIH: Sistema de Informações Hospitalares.
O maior número de informações relativas ao registro de natimortos no SIH pode ser justificado pela quantidade de gestações interrompidas que não geram DO, mas geram AIH. Parece haver maior preocupação quanto ao correto preenchimento da $\mathrm{AIH}$, instrumento de cobrança financeira, e a consequente alimentação do SIH, em detrimento do SIM, provavelmente por este sistema não gerar transferência de recursos.

Contudo, inúmeras situações que dificultam o relacionamento probabilístico e contribuem para o sub-registro foram também identificadas tanto no SIH como no SIM: informações incompletas/ausentes em campos de identificação do paciente; do médico assistente; do feto/recémnascido; da data da ocorrência do evento (período de internação e data do óbito); duplicidade de registro e simultaneidade de informações de "nascido vivo" e "nascido morto".

A má qualidade das informações aqui observadas ocorre em todos os níveis: médicos que preenchem de forma incorreta as DOs; negligência das unidades administrativas responsáveis pelo processamento das DOs e das AIHs; equívocos de digitação e ausência de envio das informações pelos municípios para o nível central do SIM no estado.

A frequente omissão da idade gestacional na DO e a subenumeração de natimortos e óbitos neonatais também constituíram limitações do SIM, tal como descrito em estudos referentes às regiões Norte e Nordeste 18. Outra importante questão a ser considerada é a completa ausência de registro de natimortos e óbitos infantis em ambos os sistemas em vários municípios, na sua maioria de pequeno porte, o que pode sugerir falha grave na gestão destes sistemas em tais localidades.

Esta situação deve ser um fator determinante no valor relativamente baixo do CMI encontrado neste estudo que foi de 16,4 por mil nascidos vivos segundo o SIM e de 18,2 por mil nascidos vivos quando considerados os registros de óbitos do SIH que não constavam o SIM. Este estudo encontra respaldo no estudo de Mello-Jorge et al. 19, que referem que coeficientes calculados utilizando os sistemas SIM/SIH podem não representar o valor real da mortalidade infantil devido às precariedades dos sistemas.

Pesquisa que utilizou dados do SIH para estimar o número de nascidos vivos, natimortos e óbitos neonatais precoces e tardios em alguns estados brasileiros em 1995 identificou que alguns estados das regiões Norte e Nordeste (como Maranhão, Ceará e Paraíba), apresentavam maior número de registros de natimortos e de óbitos neonatais no SIH que no SIM 4. 
Identificou-se o mesmo cenário nesta pesquisa, pois se observou que em 36 municípios havia mais registros de natimortos e em 12 mais registros de óbitos infantis no SIH do que no SIM. Supõe-se que possa haver maior negligência dos profissionais de saúde diante do natimorto, talvez motivada pela injustificada aceitação de que "nada mais pudesse ser feito", ou pela constatação de que eventual interrupção precoce da gravidez "seja incompatível com a vida”, daí decorrendo a negligência do registro.

Alguns autores referem que a deficiência no preenchimento da declaração de óbito por parte dos médicos poderia estar relacionada a pouca ênfase dada aos alunos na graduação, quer por pouca qualificação dada ao documento, quer por desconhecimento de sua utilidade 20,21.

Outra possível causa para o sub-registro é que em alguns municípios do Maranhão, a exigência legal do atestado de óbito para o enterro não é considerada.

Autores chamam a atenção para o fato de que o SIH não é suficiente para a investigação das causas de todos os óbitos (e esclarecimento de causa mal definida), porque parte deles ocorre em hospitais não vinculados ao SUS e consideram essa limitação intransponível, considerando que o SIH é a única grande base de dados hospitalares existente 22 . Esta se constitui também neste trabalho uma importante limitação, pois os óbitos que ocorrem na rede privada não são objetos do SIH e, portanto, não foram estudados.

Estudos com outros sistemas como o SINASC podem eventualmente ser realizados com o intuito de conhecer mais características justifiquem o sub-registro do SIM. Trevisan et al. 23 e Rodrigues 24 referem que a utilização da linkage de banco de dados SIM/SINASC permite estudar fatores de risco para óbitos neonatais e avaliar a influência do peso, fatores sociodemográficos e assistenciais na mortalidade neonatal. Entretanto, vale ressaltar que o SINASC também apresenta falhas no preenchimento e na cobertura, havendo relatos de cobertura de 91,7\% em 2006 (Ministério da Saúde. http:// www.saude.gov.br, acessado em 18/Fev/2011).

Se por um lado o relacionamento probabilístico aqui utilizado mostrou-se um facilitador na recuperação de dados, por outro, deve-se enfatizar que o adequado preenchimento da DO e da $\mathrm{AIH}$, ainda distante, deve ser um objetivo a ser perseguido para que se consiga a desejada confiabilidade integral dos dados. Supõe-se que a baixa concordância entre DO e AIH pode se dever aos diferentes princípios e finalidades de tais documentos e se faz necessário sensibilizar gestores e profissionais envolvidos sobre a importância das informações e qualidade dos dados, de modo a permitir a recuperação mútua e automática de dados.

Constituiu ponto forte deste estudo a utilização de técnica que permitiu a recuperação de dados de sub-registro de óbitos em todo o Estado do Maranhão. Por outro lado, a inexistência/dualidade de informações de natimortos e a ausência da data do óbito da criança nas AIHs das parturientes constituem-se potenciais vieses neste estudo, que podem gerar coeficientes que não refletem a realidade. Além disso, a AIH, apesar de apresentar agilidade para a disponibilização de informações, não é padrão-ouro para o cálculo de sub-registro, pois não permite obter informações detalhadas de cada caso, devido à sua própria natureza e função.

A redistribuição dos óbitos diante do preenchimento simultâneo dos campos para nascido vivo e nascido morto da AIH, para recuperação de registros de óbitos, provavelmente subestimou o CMI. Embora estes registros tenham sidos recuperados, o CMI para o Estado se manteve aquém do esperado ${ }^{3}$. Tal resultado evidencia a necessidade de maior atenção no preenchimento das informações da AIH.

Em síntese, demonstrou-se que a qualidade das informações é afetada por inconsistências no preenchimento e no fluxo, tanto do SIM quanto do SIH. Tal contexto reflete deficiências na gestão local dos sistemas. O relacionamento probabilístico apresentou-se como bom recurso de recuperação de dados para o SIM a partir do SIH. A identificação dos pontos fracos aqui apresentados facilita a intervenção dos gestores nas unidades geradoras para melhorar a qualidade das informações contribuindo para o conhecimento da realidade e possibilitando adequada intervenção das políticas de saúde pública. 


\section{Resumo}

Neste estudo utilizou-se método do relacionamento probabilístico de registros para estimar o sub-registro das informações sobre natimortos e óbitos infantis no Estado do Maranhão, Nordeste do Brasil, em 2008. Relacionaram-se os registros do Sistema de Informações Hospitalares $(\mathrm{SIH})(\mathrm{N}=374.418)$ e do Sistema de Informações de Mortalidade (SIM) ( $N=26.597), \mathrm{com}$ utilização de softwares para extração, identificação e processamento dos dados. Observou-se sub-registro de 9,7\% de natimortos, 12,0\% de óbitos neonatais e 5,3\% de pós-neonatais. Nos municípios maiores a correção do coeficiente de mortalidade infantil foi superior a 19\%. A superioridade de informações de óbitos infantis do SIH foi de 6,5\% (municípios < 25 mil habitantes), 2,0\% (municípios de 25-80 mil habitantes) e 8,3\% (municípios $>80$ mil habitantes). As inconsistências ocorreram por não preenchimento de variáveis, informações discordantes para um mesmo evento e ausência completa de registro do óbito. O método utilizado mostrou-se útil para recuperação de dados de óbitos para o SIM a partir do SIH.

Sub-Registro; Sistemas de Informação; Mortalidade

\section{Colaboradores}

Todos os autores elaboraram e revisaram o artigo.

\section{Agradecimentos}

Os autores são gratos à Fundação de Amparo à Pesquisa e ao Desenvolvimento Tecnológico do Estado do Maranhão e ao SUS (PPSUS), pelo apoio financeiro (Proc. FAPEMA/SUS no. 089_9228920); à Profa. Katia Vergheti Bloch, pela revisão deste manuscrito e, à Secretaria de Estado da Saúde do Maranhão, pela autorização do acesso ao banco de dados do SIH e do SIM.

\section{Referências}

1. Moya J, Risi Junior JB, Martinello A, Bandarra E, Bueno H, Morais Neto OL, organizadores. Salas de Situação em Saúde: compartilhando as experiências do Brasil. Brasília: Organização Pan-Americana da Saúde/Ministério da Saúde; 2010.

2. Ribeiro VS, Silva AAM. Tendências da mortalidade neonatal em São Luís, Maranhão, Brasil, de 1979 a 1996. Cad Saúde Pública 2000; 16:429-38.

3. Organização Pan-Americana da Saúde/Ministério da Saúde. IDB 2009 Brasil - indicadores e dados básicos para a saúde. Rio de Janeiro: Organização PanAmericana da Saúde/Ministério da Saúde; 2010.

4. Schramm JMA, Szwarcwald CL. Sistema hospitalar como fonte de informações para estimar a mortalidade neonatal e a natimortalidade. Rev Saúde Pública 2000; 34:272-9.

5. Mello-Jorge MHP. Sub-registro dos eventos vitais. Rev Saúde Pública 1983; 17:148-51.
6. Feijó MCC, Portela MC. Variação no custo de internações hospitalares por lesões: os casos dos traumatismos cranianos e acidentes por armas de fogo. Cad Saúde Pública 2001; 17:627-37.

7. Escosteguy CC, Portela MC, Medronho RA, Vasconcellos MTL. O Sistema de Informações Hospitalares e a assistência ao infarto agudo do miocárdio. Rev Saúde Pública 2002; 36:491-9.

8. Mendes ACG, Silva Jr. JB, Medeiros KR, Lyra TM, Melo Filho DA, Sá DA. Avaliação do Sistema de Informações Hospitalares - SIH/SUS como fonte complementar na vigilância e monitoramento de doenças de notificação compulsória. Inf Epidemiol SUS 2000; 9:67-86.

9. Ortiz LP, Oushiro DA. Perfil da mortalidade neonatal no Estado de São Paulo. São Paulo Perspect 2008; 22:19-29. 
10. Silva CF, Leite AJM, Almeida NMGS. Linkage entre bancos de dados de nascidos vivos e óbitos infantis em município do Nordeste do Brasil: qualidade dos sistemas de informação. Cad Saúde Pública 2009; 25:1552-8.

11. Drumond EF, Machado CJ, Linkage entre registros do SIHSUS e SINASC: possíveis vieses decorrentes do não-pareamento. Rev Bras Estud Popul 2007; 25:191-4.

12. Drumond EF, Machado CJ, França E. Subnotificação de nascidos vivos: procedimentos de mensuração a partir do sistema de informação hospitalar. Rev Saúde Pública 2008; 42:55-63.

13. Camargo Jr. KR, Coeli CM. Reclink 3: nova versão do programa que implementa a técnica de associação probabilística de registros (probabilistic record linkage). Cad Saúde Coletiva (Rio J) 2006; 14:399-404.

14. Mello Jorge MHP, Gotlieb SLD, Oliveira H. O Sistema de Informações sobre Nascidos Vivos: primeira avaliação dos dados brasileiros. Inf Epidemiol SUS 1996; 5:15-48.

15. Camargo Jr. KR, Coeli CM. Reclink: aplicativo para o relacionamento de bases de dados, implementando o método probabilistic record linkage. Cad Saúde Pública 2000; 16:439-47.

16. Pereira MG. Epidemiologia: teoria e prática. Rio de Janeiro: Editora Guanabara Koogan; 2007.

17. Szwarcwald CL, Leal MC, Andrade CLT, Souza Jr. PRB. Estimação da mortalidade infantil no Brasil: o que dizem as informações sobre óbitos e nascimentos do Ministério da Saúde? Cad Saúde Pública 2002; 18:1725-36.

18. França E, Lansky S. Mortalidade infantil neonatal no Brasil: situação, tendências e perspectiva. http://www.ripsa.org.br (acessado em 17/ Dez/2009).
19. Mello-Jorge MHP, Laurenti R, Gotlieb SLD. Análise da qualidade das estatísticas vitais brasileiras: a experiência de implantação do SIM e SINASC. Ciênc Saúde Coletiva 2007; 12:643-54.

20. Novaes HMD, Almeida MF, Ortiz LP. Projeto informação para tomadores de decisão em saúde pública. Tema V: gestão para redução da mortalidade infantil. 2a Ed. São Paulo: Organização Pan-Americana da Saúde/Ministério da Saúde; 2004.

21. Sarinho SW, Coutinho SB, Acioli TML. Mortalidade neonatal em Recife, Pernambuco, 1998: causas básicas e grau de conhecimento dos neonatologistas acerca do preenchimento das Declarações de Óbito. Pediatria (São Paulo) 2001; 23:279-84.

22. Teixeira CLS, Bloch KV, Klein CH, Coeli CM. Método de relacionamento de bancos de dados do Sistema de Informações sobre Mortalidade (SIM) e das Autorizações de Internação Hospitalar (BDAIH) no Sistema Único de Saúde (SUS), na investigação de óbitos de causa mal-definida no estado do Rio de Janeiro, Brasil, 1998. Epidemiol Serv Saúde 1998; 15:47-57.

23. Trevisan MR, De Lorenzi DRS, Araújo NM. Perfil da assistência pré-natal entre usuárias do Sistema Único de Saúde em Caxias do Sul. Rev Bras Ginecol Obstet 2002; 24:293-9.

24. Rodrigues EM. Estimativas de riscos de mortalidade neonatal a partir de dados das declarações de nascidos vivos e óbitos: estado de São Paulo, Brasil [Tese de Doutorado]. São Paulo: Escola Paulista de Medicina, Universidade Federal de São Paulo; 2003.

Recebido em 26/Nov/2010

Versão final reapresentada em 10/Mai/2011

Aprovado em 18/Mai/2011 